VERSITA

\author{
TATRA \\ MOUNTaiNs \\ Mathematical Publications \\ DOI: $10.2478 /$ v10127-011-0003-7 \\ Tatra Mt. Math. Publ. 48 (2011), 25-40
}

\title{
KERNEL-RESOLVENT RELATIONS FOR AN INTEGRAL EQUATION
}

\author{
TheOdore A. Burton
}

\begin{abstract}
We consider a scalar integral equation $z(t)=a(t)-\int_{0}^{t} C(t, s)[z(s)+$ $G(s, z(s))] d s$ where $|G(t, z)| \leq \phi(t)|z|, C$ is convex, and $a \in\left(L^{\infty} \cap L^{2}\right)[0, \infty)$. Related to this is the linear equation $x(t)=a(t)-\int_{0}^{t} C(t, s) x(s) d s$ and the resolvent equation $R(t, s)=C(t, s)-\int_{s}^{t} C(t, u) R(u, s) d u$. A Liapunov functional is constructed which gives qualitative results about all three equations. We have two goals. First, we are interested in conditions under which properties of $C$ are transferred into properties of the resolvent $R$ which is used in the variation-of-parameters formula. We establish conditions on $C$ and functions $b$ so that $\int_{0}^{t} C(t, s) b(s) d s \rightarrow 0$ as $t \rightarrow \infty$ and is in $L^{2}[0, \infty)$ implies that $\int_{0}^{t} R(t, s) b(s) d s \rightarrow 0$ as $t \rightarrow \infty$ and is in $L^{2}[0, \infty)$. Such results are fundamental in proving that the solution $z$ satisfies $z(t) \rightarrow a(t)$ as $t \rightarrow \infty$ and that $\int_{0}^{\infty}(z(t)-a(t))^{2} d t<\infty$.
\end{abstract}

This is in final form and no other version will be submitted.

\section{Introduction}

There are many important relations between the kernel, $C(t, s)$, of the integral equation

$$
x(t)=a(t)-\int_{0}^{t} C(t, s) x(s) d s,
$$

where $a$ is continuous for $t \geq 0$ and $C$ is continuous for $0 \leq s \leq t<\infty$, and the resolvent, $R(t, s)$, solving

$$
R(t, s)=C(t, s)-\int_{s}^{t} C(t, u) R(u, s) d u .
$$

The function $R$ is used in the variation-of-parameters formula

(C) 2011 Mathematical Institute, Slovak Academy of Sciences.

2010 Mathematics Subject Classification: 47G05, 34D20.

Keywords: integral equations, Liapunov functionals. 


$$
x(t)=a(t)-\int_{0}^{t} R(t, s) a(s) d s .
$$

However, three such relations stand out for their simplicity and utility. First, if there is an $\alpha<1$ with

$$
\sup _{0 \leq t<\infty} \int_{0}^{t}|C(t, s)| d s \leq \alpha,
$$

then

$$
\sup _{0 \leq t<\infty} \int_{0}^{t}|R(t, s)| d s \leq \frac{\alpha}{(1-\alpha)} .
$$

It is an old result and its origin seems to have been lost. Among its many applications is the immediate conclusion that $a \in L^{\infty}[0, \infty)$ implies $x \in L^{\infty}[0, \infty)$ [2, p. 54], where $x$ solves (1).

Next, if there is a $\beta<1$ with

$$
\sup _{0 \leq s \leq t<\infty} \int_{s}^{t}|C(u, s)| d u \leq \beta,
$$

then

$$
\sup _{0 \leq s \leq t<\infty} \int_{s}^{t}|R(u, s)| d u \leq \beta /(1-\beta)
$$

yielding immediate $L^{p}[0, \infty)$ results for $x$. It was first proved in [2, p. 54].

Notice that the second coordinate of $C$ may be thought of as the $L^{\infty}$ coordinate, while the first is the $L^{p}$ coordinate, properties lost in the convolution case.

Finally, we come to a relation which will be modified for this project. It was obtained by Strauss [9]. If (4) holds and if for each $T>0$ we have

$$
\lim _{t \rightarrow \infty} \int_{0}^{T}|C(t, s)| d s=0
$$

then

$$
\lim _{t \rightarrow \infty} \int_{0}^{T}|R(t, s)| d s=0
$$

with immediate application to the nonlinear perturbed equation

$$
z(t)=a(t)-\int_{0}^{t} C(t, s)[z(s)+G(s, z(s))] d s
$$




\section{KERNEL-RESOLVENT RELATIONS FOR AN INTEGRAL EQUATION}

with $a$ and $C$ as in (1) and $G$ continuous for $0 \leq t<\infty$ and $z \in \Re$. Now (10) is decomposed into (1) and

$$
z(t)=x(t)-\int_{0}^{t} R(t, s) G(s, z(s)) d s
$$

with $x$ the solution of (1) and $R$ the solution of (3).

We call the pairs (4) and (5), (6) and (7), (8) and (9) (with (4)) transfer principles. In this paper we focus on new ones related to real-world problems and which avoid the smallness conditions. In joint work with $\mathrm{D}$ w ig g in s ([4], [5]) we removed (4) in the (8)-(9) set, replacing it with a global Lipschitz condition on $R$ with respect to $s$ and applying the result to (11). The general problem of (10) and (11) is discussed in Strauss [9, Miller 6], Miller-Nohel-

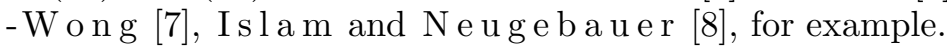

In all of that work there are difficulties which seem to stem from a step in the work, where we take the absolute value inside the integral and consider $\int_{0}^{t}|R(t, s) G(s, z(s))| d s$. The present study differs in that we study that term without ever taking an absolute value. This results in reduced assumptions and, especially, we never require either (4) or (6). It will turn out that $C$, itself, can be arbitrarily large and so can various integrals of $C$. This is a significant contrast to the three aforementioned relations. The result will enable us to say that $z(t) \rightarrow a(t)$ as $t \rightarrow \infty$ and that $\int_{0}^{\infty}(z(t)-a(t))^{2} d t<\infty$.

In this paper we will extend this list of relations between $C$ and $R$ for the case in which (8) holds and $C$ is convex,

$$
C(t, s) \geq 0, C_{s}(t, s) \geq 0, C_{t}(t, s) \leq 0, C_{s t}(t, s) \leq 0 \quad \text { for } \quad 0 \leq s \leq t<\infty,
$$

as well as present a detailed analysis of (10) and (11).

The work is centered on (10) with two different assumptions on $G$ which illustrate the range of application of the ideas. First, we assume that

$$
|G(t, z)| \leq \phi(t)|z|, \quad \phi \in L^{1}[0, \infty) .
$$

In 1928 Volterra [10] noted that many real-world problems were being modelled by integral and integro-differential equations with convex kernels. In some cases, such as viscoelasticity, convexity has been deduced from first principles, in other problems the model is descriptive. In the heat equation there is convexity in the kernel for $t \neq s$ and it has been shown in Burton [3] that such singularities do not materially affect the behavior of solutions. Subjectively, investigators have chosen convexity because it yields behavior coinciding with our intuitive idea of fading memory.

The overwhelming reason for our preference for a convex kernel comes from the fact that Liapunov functionals allow us to use arbitrarily large kernels and avoid the Draconian conditions found in (4) and (6). 


\section{THEODORE A. BURTON}

\section{Strategy}

In (10), $G(t, z)$ is considered to be a small perturbation of $x$. The task is to find significant properties of the solution, $x$, of (1), determine reasoned assumptions on $G(t, z)$, and prove that $z$ is closely related to $x$. In this section we will illustrate one way in which that can be done very well under the assumption that $C$ is convex. The work proceeds in three steps.

Step 1. For $C$ convex, a suggestion of Volterra in 1928 led to a Liapunov functional for a nonlinear form of (1) which leads to fine details about the solution of (1). The linear form of that Liapunov function is (see [2, p. 131] or [1] for the construction)

$$
V(t)=\int_{0}^{t} C_{s}(t, s)\left(\int_{s}^{t} x(u) d u\right)^{2} d s+C(t, 0)\left(\int_{0}^{t} x(u) d u\right)^{2}
$$

having derivative (as shown in the appendix) satisfying

$$
V^{\prime}(t) \leq a^{2}(t)-x^{2}(t)-(x(t)-a(t))^{2} .
$$

In addition, if there is a $B>0$ with $C(t, t) \leq B$, then

$$
(x(t)-a(t))^{2} \leq 2 B V(t) .
$$

Thus,

$$
a \in L^{2}[0, \infty) \Longrightarrow x \in L^{2}[0, \infty), x-a \in L^{2}[0, \infty)
$$

and if, in addition,

$$
C(t, t) \leq B \quad \text { and } \quad a \in L^{\infty}, \quad \text { then } \quad x \in L^{\infty} .
$$

If we also assume (8), then we can conclude that $x(t) \rightarrow a(t)$ as $t \rightarrow \infty$. The three assumptions of $a \in L^{2}, C$ convex, and (8) leave us with very extensive information about $x$.

Step 2. As $G$ is a small perturbation, we expect to prove that

$$
z \in L^{2} \cap L^{\infty}, \quad z-x \in L^{2}[0, \infty), \quad z(t) \rightarrow x(t) .
$$

In the next section we will find conditions under which $z \in\left(L^{\infty} \cap L^{2}\right)[0, \infty)$. Those are, so to speak, the "coarse" properties of $z$. Here, we will assume those properties and use that information to find the "finer" property of $z(t) \rightarrow x(t)$ as $t \rightarrow \infty$ and $z-x \in L^{2}[0, \infty)$. Next, as $G$ is a small perturbation for a linear equation we would ask that

$$
|G(t, z)| \leq \phi(t)|z|
$$

and ponder the assumptions for $\phi$. 


\section{KERNEL-RESOLVENT RELATIONS FOR AN INTEGRAL EQUATION}

We note that when $z \in L^{\infty}$, then (11) can be considered as

where

$$
z(t)=x(t)-\int_{0}^{t} R(t, s) b(s) d s
$$

$$
|b(s)| \leq K \phi(s), \quad K \text { is a bound on }|z| \text {. }
$$

This is precisely what was done in the corresponding investigation of ordinary differential equations. From our conjecture about the properties of $z$, our goal is to prove

$$
f(t):=\int_{0}^{t} R(t, s) b(s) d s \in L^{2}[0, \infty) \text { and } \int_{0}^{t} R(t, s) b(s) d s \rightarrow 0 .
$$

This will lead us directly to the assumption on $\phi$.

Step 3. Since we know how to find the most intricate properties of the solution of (1), we will construct a linear equation containing $f$ as its solution. Using that we will show that (13) holds, concluding then from (10) that

$$
z-x \in L^{2}[0, \infty) \text { and } z(t) \rightarrow x(t) \rightarrow a(t) \quad \text { as } t \rightarrow \infty .
$$

More importantly, we will have discovered how to parlay the properties of $\int_{0}^{t} C(t, s) b(s) d s$ into the properties of $R$ listed in (13) whenever $C$ is convex and $(8)$ holds, extending the pairs of properties listed in the introduction without asking smallness conditions on $C$. Here are the details for constructing (13).

Let $b:[0, \infty) \rightarrow \Re$ be continuous, write $(2)$ as

and then form

$$
R(t, s) b(s)=C(t, s) b(s)-\int_{s}^{t} C(t, u) R(u, s) d u b(s),
$$

$$
\begin{aligned}
\int_{0}^{t} R(t, s) b(s) d s & =\int_{0}^{t} C(t, s) b(s) d s-\int_{0}^{t} \int_{s}^{t} C(t, u) R(u, s) d u b(s) d s \\
& =\int_{0}^{t} C(t, s) b(s) d s-\int_{0}^{t} C(t, u) \int_{0}^{u} R(u, s) b(s) d s d u
\end{aligned}
$$

which we will write as

$$
f(t)=S(t)-\int_{0}^{t} C(t, u) f(u) d u
$$

with

$$
f(t)=\int_{0}^{t} R(t, s) b(s) d s, \quad S(t)=\int_{0}^{t} C(t, s) b(s) d s .
$$




\section{THEODORE A. BURTON}

We define

$$
V(t)=\int_{0}^{t} C_{s}(t, s)\left(\int_{s}^{t} f(u) d u\right)^{2} d s+C(t, 0)\left(\int_{0}^{t} f(u) d u\right)^{2}
$$

and obtain, under the convexity assumption on $C$, that

$$
V^{\prime}(t) \leq-(S(t)-f(t))^{2}-f^{2}(t)+S^{2}(t),
$$

as it is seen in the appendix. In addition, in the appendix we see that when

$$
C(t, t) \leq B
$$

then

$$
(S(t)-f(t))^{2} \leq 2 B V(t) .
$$

Thus, (17) and (19) yield the following result with exact counterpart for (1).

TheOREM 2.1. If $C$ is convex, if $C(t, t) \leq B$, and if $S \in L^{2}[0, \infty)$, then

$$
\frac{1}{2 B}(S(t)-f(t))^{2}+\int_{0}^{t}(S(u)-f(u))^{2} d u+\int_{0}^{t} f^{2}(u) d u \leq \int_{0}^{\infty} S^{2}(u) d u .
$$

This theorem gives us, in a simple way, a relation between $C$ and $R$. The relation is also found in Burton-D w ig g ins [5], but was not used in the way we use it here; instead, we worked with the absolute value of $R$, a technique which we studiously avoid here.

TheOREM 2.2. Let $C$ be convex and suppose that $\int_{0}^{t} C(t, s) h(s) d s \rightarrow 0$ as $t \rightarrow \infty$ for every continuous function $h:[0, \infty) \rightarrow \Re$ with $h \in L^{2}[0, \infty)$. If $b:[0, \infty) \rightarrow \Re$ is continuous and if $S(t) \in L^{2}[0, \infty)$ and $S(t) \rightarrow 0$ as $t \rightarrow \infty$, then the same is true for $f$; here, $b, S$, and $f$ satisfy (15).

Proof. As $C$ is convex, (17) holds. As $S \in L^{2}[0, \infty)$, the same is true for $f$ from (17). As $f \in L^{2}[0, \infty)$ it qualifies as the $h$ of the theorem and so $\int_{0}^{t} C(t, s) f(s) d s \rightarrow 0$ as $t \rightarrow \infty$. Then from (14) we see that $f(t)-S(t) \rightarrow 0$ as $t \rightarrow \infty$. As $S(t) \rightarrow 0$ so does $f$. This completes the proof.

Sequence of steps. In all of this, everything starts with the given $C(t, s)$ being convex. Next, with our Liapunov functional in mind, $b(t)$ must be tentatively selected from the vector space of functions for which $S \in L^{2}[0, \infty)$. Automatically, $f \in L^{2}[0, \infty)$. Now, the vector space from which $b$ is drawn must be refined so that $S(t) \rightarrow 0$ as $t \rightarrow \infty$; here, (8) will play a central role. Finally, we must show that $f(t)-S(t) \rightarrow 0$ as $t \rightarrow \infty$ and, again, that it will be a simple consequence of (8). In solving (10) we will have $z(t)-a(t) \in L^{2}[0, \infty)$ and $z(t) \rightarrow a(t)$ as $t \rightarrow \infty$. That will be very strong convergence. In the context of this problem, $b(t)=G(t, z(t))$ and $|b(t)| \leq \phi(t)|z(t)|$; then our assumptions on $\phi$ are based 


\section{KERNEL-RESOLVENT RELATIONS FOR AN INTEGRAL EQUATION}

on reason, not technical necessity. There is a continuum of such functions, $b$, and a subclass is given in B u r t o n-D w i g g in s [5]. Two subclasses are simple and of considerable interest; these are $b \in L^{1}[0, \infty)$ and $b \in L^{2}[0, \infty)$. We will focus on them after we have assured ourselves that $z$ really does satisfy the conjectured properties of $z \in\left(L^{\infty} \cap L^{2}\right)$. Thus, we must pause here to obtain those properties of $z$.

\section{The Liapunov functional}

We will use our Liapunov functional directly on (10), examine the derivative and deduce from that a set of conditions needed to have $z \in L^{2}[0, \infty)$ and $z \in L^{\infty}$. Thus, the conditions which appear in the theorem below are selected from the various steps of the proof.

Theorem 3.1. Let $C$ be convex, $C(t, t) \leq B$ for some $B>0$, and let (12) hold with

$$
a \in\left(L^{\infty} \cap L^{2}\right)[0, \infty), \quad \phi \in\left(L^{1} \cap L^{2}\right)[0, \infty),
$$

where $a$ is from (1) and $\phi$ is introduced in (12). If $x$ solves (1), if $z$ solves (10), and if $R$ solves (3), then

$$
x, z, \quad \text { and } \quad \int_{0}^{t} R(t, s) G(s, z(s)) d s \in\left(L^{\infty} \cap L^{2}\right)[0, \infty),
$$

while $G(t, z) \in\left(L^{1} \cap L^{2}\right)[0, \infty)$.

P r o of. Define a Liapunov functional for (10) by

$$
\begin{aligned}
V(t)= & \int_{0}^{t} C_{s}(t, s)\left(\int_{s}^{t}[z(u)+G(u, z(u))] d u\right)^{2} d s \\
& +C(t, 0)\left(\int_{0}^{t}[z(u)+G(u, z(u))] d u\right)^{2} .
\end{aligned}
$$

In the appendix we show that if $C(t, t) \leq B$ for some $B>0$, then

$$
(z(t)-a(t))^{2} \leq 2 B V(t)
$$

and that the derivative of $V$ along a solution of (10) satisfies

$$
\begin{aligned}
V^{\prime}(t) & \leq 2[z+G(t, z)][a(t)-z(t)] \\
& =2\left[z a(t)-z^{2}+a(t) G(t, z)-z G(t, z)\right] .
\end{aligned}
$$


Use (12) and note that for any $\epsilon \in\left(0, \frac{1}{2}\right)$ we can find $M>0$ with

$$
V^{\prime}(t) \leq 2\left[\epsilon z^{2}+M a^{2}(t)-z^{2}+\epsilon \phi^{2}(t) z^{2}+M a^{2}(t)+\phi z^{2}\right] .
$$

Thus,

$$
V^{\prime}(t) \leq 2\left[(-1+\epsilon) z^{2}+2 M a^{2}(t)+\left(\phi+\epsilon \phi^{2}(t)\right) z^{2}\right]
$$

We proceed in two steps, first showing $z \in L^{\infty}$ and then $z \in L^{2}[0, \infty)$. From (25) we have

$$
V^{\prime}(t) \leq 4 M a^{2}(t)+2\left(\phi(t)+\epsilon \phi^{2}(t)\right) z^{2}(t)
$$

and we note the algebraic relation $(z-a)^{2} \geq\left(\frac{1}{2}\right) z^{2}-a^{2}$ so that from $(z-a)^{2} \leq$ $2 B V$ we obtain

or

$$
\left(\frac{1}{2}\right) z^{2}-a^{2} \leq 2 B V
$$

$$
z^{2} \leq 4 B V+2 a^{2}
$$

so

$$
V^{\prime}(t) \leq 4 M a^{2}(t)+2\left(\phi(t)+\epsilon \phi^{2}(t)\right) 4 B V(t)+4\left(\phi(t)+\epsilon \phi^{2}(t)\right) a^{2}(t) .
$$

By (21) we can find $\gamma, \mu \in L^{1}[0, \infty)$ with

$$
V^{\prime}(t) \leq \gamma(t)+\mu(t) V(t)
$$

yielding $V \in L^{\infty}[0, \infty)$ and, because $a \in L^{\infty}$, so is $z$.

Going back to $(25)$ with $z \in L^{\infty}[0, \infty)$, we have $\left(\phi+\epsilon \phi^{2}\right) z^{2} \in L^{1}[0, \infty)$ so now an integration of (25) yields $z \in L^{2}[0, \infty)$. We showed the same for $x$ in Section 2. These in (11) yield

$$
\int_{0}^{t} R(t, s) G(s, z(s)) d s \in\left(L^{\infty} \cap L^{2}\right)[0, \infty),
$$

as well as $G(t, z) \in\left(L^{1} \cap L^{2}\right)[0, \infty)$.

\section{An explicit kernel-resolvent relation}

Everything centers around Theorem 2.2. We must have

$$
S(t)=\int_{0}^{t} C(t, s) b(s) d s \in L^{2}[0, \infty)
$$

where $b(t)$ majorizes $G(t, z)$ with $|G(t, z)| \leq \phi(t)|z|$ when we have $z \in\left(L^{2} \cap L^{\infty}\right)$. In short, given $C(t, s)$, what are our choices for $\phi$ ? There is a whole continuum 
of choices which lie between two prominent branches. First, we can start with $b \in L^{1}[0, \infty)$ when $C$ is, roughly, in $L^{2}$. Another branch is found by taking $b \in L^{2}[0, \infty)$ and, again roughly, $C \in L^{1}$. We can then fill in between the branches using Hölder's inequality.

Here is the first branch.

Lemma 4.1. Let $b(t)$ be continuous, $\int_{0}^{\infty}|b(s)| d s=: M<\infty$, and let

$$
\sup _{0 \leq s \leq t<\infty} \int_{s}^{t} C^{2}(u, s) d u=: J<\infty .
$$

Then $S(t)=\int_{0}^{t} C(t, s) b(s) d s \in L^{2}[0, \infty)$.

Proof. Let

$$
L(t):=\int_{0}^{t}\left(\int_{0}^{u} C(u, s) b(s) d s\right)^{2} d u
$$

so that

$$
\begin{aligned}
L(t) & \leq \int_{0}^{t} \int_{0}^{u}|b(s)| d s \int_{0}^{u} C^{2}(u, s)|b(s)| d s d u \\
& \leq M \iint_{0}^{t} C^{2}(u, s)|b(s)| d s d u \\
& =M \int_{0}^{t} \int_{s}^{t} C^{2}(u, s) d u|b(s)| d s \\
& \leq M J \int_{0}^{t}|b(s)| d s \leq J M^{2}
\end{aligned}
$$

as required.

Given $C$ satisfying the above conditions, we choose $b$ as stated and we can be sure that $S$ and $f$ are in $L^{2}[0, \infty)$. Next, we want to be sure that $f(t)=$ $\int_{0}^{t} R(t, s) b(s) d s \rightarrow 0$ as $t \rightarrow \infty$. We ensure that in three steps.

Notation. The symbol

$$
\|C\|=\sup _{0 \leq s \leq t<\infty}|C(t, s)| \text { and }\|b\|^{[0, T]}=\sup _{0 \leq s \leq T}|b(s)| .
$$

Definition 4.2. A function $C(t, s)$ is said to "tend to zero on-average" as $t \rightarrow \infty$ if it is bounded for $0 \leq s \leq t<\infty$ and if for each $T>0$, then (8) holds. 
Lemma 4.3. Let $\sup _{t \geq 0} \int_{0}^{t} C^{2}(t, s) d s=: M^{2}<\infty$ and let $C(t, s)$ tend to zero on-average as $t \rightarrow \infty$. If $S \in L^{2}[0, \infty)$ and $f \in L^{2}[0, \infty)$, then for (14) we have

$$
f(t)-S(t) \rightarrow 0 \quad \text { as } t \rightarrow \infty \text {. }
$$

Proof. Let $\int_{0}^{\infty} f^{2}(u) d u=: H^{2},\|C\|=: N^{2}$, and $\int_{0}^{\infty} S^{2}(t) d t=: P^{2}$. For $0<T<t$ we have

$$
\begin{aligned}
|f(t)-S(t)| & \leq \int_{0}^{t}|C(t, s) f(s)| d s \\
& =\int_{0}^{T}|C(t, s) f(s)| d s+\int_{T}^{t}|C(t, s) f(s)| d s \\
& \leq \sqrt{\int_{0}^{T} f^{2}(s) d s \int_{0}^{T} C^{2}(t, s) d s}+\sqrt{\int_{T}^{t} C^{2}(t, s) d s \int_{T}^{t} f^{2}(s) d s} \\
& \leq H N \sqrt{\int_{0}^{T}|C(t, s)| d s}+M \sqrt{\int_{T}^{\infty} f^{2}(s) d s .}
\end{aligned}
$$

For a given $\epsilon>0$, take $T$ so large that the last term is less than $\frac{\epsilon}{2}$. Then take $t$ so large that the next-to-last term is less than $\frac{\epsilon}{2}$.

Lemma 4.4. If $C(t, s)$ tends to zero on-average as $t \rightarrow \infty$, if $b \in L^{1}[0, \infty)$, and if $b(t)$ is bounded on compact intervals, then $S(t) \rightarrow 0$ as $t \rightarrow \infty$.

Pro of. For $0<T<t$ we have

$$
\begin{aligned}
|S(t)| & =\left|\int_{0}^{t} C(t, s) b(s) d s\right| \\
& \leq \int_{0}^{T}|C(t, s) b(s)| d s+\int_{T}^{t}|C(t, s) b(s)| d s \\
& \leq\|b\|^{[0, T]} \int_{0}^{T}|C(t, s)| d s+\|C\| \int_{T}^{\infty}|b(s)| d s,
\end{aligned}
$$

from which the result follows. 


\section{KERNEL-RESOLVENT RELATIONS FOR AN INTEGRAL EQUATION}

Theorem 4.5. Let $b$ be continuous, let $b \in L^{1}[0, \infty)$, let $C$ be convex, let $C(t, s)$ tend to zero on-average as $t \rightarrow \infty$, let $(29)$ hold, and let $\int_{0}^{t} C^{2}(t, s) d s \in$ $L^{\infty}[0, \infty)$. Then

$$
\int_{0}^{t} C(t, s) b(s) d s \rightarrow 0 \quad \text { as } \quad t \rightarrow \infty
$$

and

$$
\int_{0}^{t} R(t, s) b(s) d s \rightarrow 0 \quad \text { as } \quad t \rightarrow \infty .
$$

P r o of. As $b \in L^{1}[0, \infty)$ and continuous, while (29) holds, by Lemma 4.1 we have $S \in L^{2}[0, \infty)$ and so by convexity $f \in L^{2}[0, \infty)$. As $C(t, s) \rightarrow 0$ on-average and $\int_{0}^{t} C^{2}(t, s) d s \in L^{\infty}$, we have by Lemma 4.3 that $f(t)-S(t) \rightarrow 0$. Since $S(t) \rightarrow 0$ by Lemma 4.4, $f(t) \rightarrow 0$, as required.

We can now offer a solution to our problem with (10).

TheOREM 4.6. Let (12) and (21) hold, let $C$ be convex, let (29) hold, let

$$
\int_{0}^{t} C^{2}(t, s) d s \in L^{\infty}[0, \infty),
$$

and let $C(t, s)$ tend to zero on-average as $t \rightarrow \infty$. If $z$ solves $(10)$, then $z(t) \rightarrow a(t)$ as $t \rightarrow \infty$ and $\int_{0}^{\infty}(z(t)-a(t))^{2} d t<\infty$.

P r o o f. As (1) and (14) have the same form, (20) translates into a result for (1) with $a \in\left(L^{2} \cap L^{\infty}\right)[0, \infty)$. Applying the technique of Lemma 4.3 to (1) yields $x \in\left(L^{2} \cap L^{\infty}\right)[0, \infty), x-a \in L^{2}[0, \infty)$, and $x(t) \rightarrow a(t)$ as $t \rightarrow \infty$.

By Theorem 3.1 we have $G(t, z) \in\left(L^{1} \cap L^{2}\right)[0, \infty)$, so we define $b(t)=$ $G(t, z(t)), f(t)=\int_{0}^{t} R(t, s) b(s) d s$, and $S(t)=\int_{0}^{t} C(t, s) b(s) d s$ forming (14). By Lemma 4.1 we have $S \in L^{2}$, so the same is true for $f$. Then by Lemma 4.3 $f(t)-S(t) \rightarrow 0$; but $b(t)$ is continuous so by Lemma $4.4, S(t) \rightarrow 0$, yielding $f(t) \rightarrow 0$. Hence, $z(t) \rightarrow x(t) \rightarrow a(t)$ as $t \rightarrow \infty$. As $f \in L^{2}$, then $z-x \in L^{2}$. It follows that $z-a \in L^{2}$. This completes the proof.

\section{A parallel result}

We turn now to a result at the other end of the continuum and work with a pair of $L^{1}$ conditions on $C$ instead of the $L^{2}$ conditions. 


\section{THEODORE A. BURTON}

LEMMA 5.1. If $b \in L^{2}[0, \infty)$ and is continuous, if there are positive constants $M$ and $P$ with $\int_{0}^{t}|C(t, s)| d s \leq M$ and $\int_{s}^{t}|C(u, s)| d u \leq P$, then $S \in L^{2}[0, \infty)$.

P r o of. We have

$$
\begin{aligned}
\int_{0}^{t} S^{2}(u) d u & =\int_{0}^{t}\left(\int_{0}^{u} C(u, s) b(s) d s\right)^{2} d u \\
& \leq \int_{0}^{t} \int_{0}^{u}|C(u, s)| d s \int_{0}^{u}|C(u, s)| b^{2}(s) d s d u \leq M \int_{0}^{t}|C(u, s)| b^{2}(s) d s d u \\
& =M \int_{0}^{t} \int_{s}^{t}|C(u, s)| b^{2}(s) d u d s=M \int_{0}^{t} \int_{s}^{t}|C(u, s)| d u b^{2}(s) d s \\
& \leq M P \int_{0}^{t} b^{2}(s) d s \leq M P \int_{0}^{\infty} b^{2}(s) d s .
\end{aligned}
$$

Lemma 5.2. If $b \in L^{2}[0, \infty)$, if for each $T>0$, then $\left\|b^{2}\right\|^{[0, T]} \in L^{\infty}$, if $\int_{0}^{t}|C(t, s)| d s \leq M$, and if $C(t, s)$ tends to zero on-average as $t \rightarrow \infty$, then $S(t) \rightarrow 0$ as $t \rightarrow \infty$.

P r o of. Note that $\|C\|<\infty$. We have

$$
\begin{aligned}
S^{2}(t)= & \left(\int_{0}^{t} C(t, s) b(s) d s\right)^{2} \\
\leq & \int_{0}^{t}|C(t, s)| d s \int_{0}^{t}|C(t, s)| b^{2}(s) d s \leq M \int_{0}^{t}|C(t, s)| b^{2}(s) d s \\
& (\text { and for } 0<T<t \text { we have }) \\
= & M\left[\int_{0}^{T}|C(t, s)| b^{2}(s) d s+\int_{T}^{t}|C(t, s)| b^{2}(s) d s\right] \\
\leq & M\left[\left\|b^{2}\right\|^{[0, T]}|C(t, s)| d s+\|C\| \int_{T}^{t} b^{2}(s) d s\right]
\end{aligned}
$$

and this will tend to zero as $t \rightarrow \infty$. 


\section{KERNEL-RESOLVENT RELATIONS FOR AN INTEGRAL EQUATION}

LEMMA 5.3. If $S \in L^{2}[0, \infty)$, if $\int_{0}^{t}|C(t, s)| d s \leq M$, if $C$ is convex, and if $C(t, s)$ tends to zero on-average (so $\|C\|$ is finite), then $f \in L^{2}[0, \infty)$, and $f(t)-S(t) \rightarrow 0$ as $t \rightarrow \infty$.

Proof. As $S \in L^{2}$ and $C$ is convex, we have $f \in L^{2}$. Also, $C \geq 0$ and $f$ is continuous. We see that

$$
(f(t)-S(t))^{2}=\left(\int_{0}^{t} C(t, s) f(s) d s\right)^{2} \leq \int_{0}^{t} C(t, s) d s \int_{0}^{t} C(t, s) f^{2}(s) d s
$$

and the rest is identical to that of Lemma 5.2.

TheOREM 5.4. Let $b$ be continuous and in $L^{2}[0, \infty)$ and let $C$ be convex and tend to zero on-average as $t \rightarrow \infty$. Suppose also that there are positive numbers $M, P$ with $\int_{0}^{t} C(t, s) d s \leq M$ and $\int_{s}^{t} C(u, s) d u \leq P$. Then $\int_{0}^{t} C(t, s) b(s) d s \in L^{2}[0, \infty)$, $\int_{0}^{t} C(t, s) b(s) d s \rightarrow 0$ as $t \rightarrow \infty$, and $\int_{0}^{t} R(t, s) b(s) d s \rightarrow 0$ as $t \rightarrow \infty$ as well as being in $L^{2}[0, \infty)$.

P r o o f. By Lemma 5.1 we have $S \in L^{2}[0, \infty)$. As $C$ is convex, we now have $f \in$ $L^{2}[0, \infty)$. As $C(t, s) \rightarrow 0$ on-average, $f(t)-S(t) \rightarrow 0$ as $t \rightarrow \infty$ by Lemma 5.3. By Lemma 5.2 we see that $S(t) \rightarrow 0$ as $t \rightarrow \infty$ and that completes the proof.

We offer a comparison between Theorem 4.5 and Theorem 5.4. In Theorem 4.5 we had $\int_{0}^{t} C^{2}(t, s) d s$ bounded and $b(t)=G(t, z(t)) \in L^{1}$. This allowed us to show that $\int_{0}^{t} R(t, s) G(s, z(s)) d s \in L^{2}$ and tends to zero so $z(t) \rightarrow x(t)$. We had to work with (11) because if we had worked directly with (10), then we would have needed $z+G(t, z) \in L^{1}$; it is very difficult to show $z \in L^{1}$ because that is known to imply a very strong kind of uniform asymptotic stability.

By contrast, Theorem 5.4 will allow us to work directly with (10). Our Theorem 3.1 yields $z+G(t, z) \in L^{2}$ so we take $b(t)=z+G(t, z)$ and assume $\int_{0}^{t} C(t, s) d s \leq M$ and $\int_{s}^{t} C(u, s) d u \leq P$ obtaining $\int_{0}^{t} C(t, s) b(s) d s \in L^{2}$ and tending to zero. That yields $z-a \in L^{2}$ and $z-a \rightarrow 0$. Of course, there are other benefits of the conclusion that $\int_{0}^{t} R(t, s) b(s) d s \rightarrow 0$ and is in $L^{2}$, as we will see below.

As examples, first let $C(t, s)=[1+t-s]^{-\frac{2}{3}}$ so that $\int_{0}^{t} C^{2}(t, s) d s$ is bounded. Then Theorem 5.4 fails and we are forced to study (11) using Theorem 4.5. Next, let $C(t, s)=[1+t-s]^{-\frac{4}{3}}$ so that $\int_{0}^{t} C(t, s) d s$ and $\int_{s}^{t} C(u, s) d u$ are bounded. We can use Theorem 5.4 on (10) directly.

Now we consider (10) with a different assumption on $\phi$, namely

$$
|G(t, z)| \leq \phi(t)|z|, \quad \phi \in L^{2}[0, \infty), \quad \phi(t) \leq \beta<1 .
$$

This is a harsh condition as it has $z$ to dominate $G(t, z)$ for every $(t, z)$. 


\section{THEODORE A. BURTON}

Define $V$ as in (23), but at (25) we now make $\epsilon$ so small (and $M$ so large) that

$$
\beta+\epsilon \beta^{2}+\epsilon \leq \mu<1
$$

Then from (25) we have

$$
V^{\prime}(t) \leq 2\left[(-1+\mu) z^{2}+2 M a^{2}(t)\right] .
$$

Accordingly, (21) is replaced by (31) and

$$
a \in\left(L^{\infty} \cap L^{2}\right)[0, \infty) .
$$

We now have a result parallel to Theorem 3.1.

TheORem 5.5. Let $C$ be convex, $C(t, t) \leq B$ for some $B>0$, and let (31) and (33) hold. If $x$ solves (1), if $z$ solves (10), and if $R$ solves (3), then

$$
x, z, G(t, z) \in\left(L^{\infty} \cap L^{2}\right)[0, \infty) .
$$

Proof. From (32) we see that $V$ is bounded, that $z$ is bounded, and that $z \in L^{2}[0, \infty)$. By $(31)$,

$$
G(t, z) \in L^{2}[0, \infty)
$$

and it is bounded. Clearly, $x \in L^{2}[0, \infty)$ and is bounded.

Recall that in the proof of Theorem 4.6 we argued that

$$
x(t)-a(t) \rightarrow 0 \text { as } t \rightarrow \infty \text { and } \quad x-a \in L^{2} .
$$

Taking $b(t)=G(t, z)$, we form

$$
f(t)=S(t)-\int_{0}^{t} C(t, s) f(s) d s .
$$

Note that $b$ is continuous. We summarize as follows.

TheORem 5.6. Let the conditions of Theorems 5.4 and 5.5 hold. Then

$$
\int_{0}^{t} R(t, s) G(s, z(s)) d s \rightarrow 0 \quad \text { as } t \rightarrow \infty \quad \text { and } \quad z-a \in L^{2}[0, \infty) .
$$

As an example, we can let $C(t, s)=[1+t-s]^{-(1+\delta)}$ for $\delta>0$ and $b(t)=$ $\phi(t)=[2+t]^{-\frac{3}{4}}$. All conditions of Lemmas 5.1, 5.2, and 5.3 hold. However, Lemma 4.5 is not satisfied. Theorem 5.6 holds, but Theorem 4.6 does not. They are essentially different results and there is a continuum of results "between" them and they are obtained using Hölder's inequality in the process of forming $S(t)$, establishing that $S \in L^{2}$. Each gives different conditions on $b$ and $C$ to ensure that $\int_{0}^{t} R(t, s) b(s) d s \in L^{2}$ and tends to zero. 


\section{Appendix}

The general nonlinear Liapunov functional (see [1]) defined by

for

$$
V(t)=\int_{0}^{t} C_{s}(t, s)\left(\int_{s}^{t} g(u, z(u)) d u\right)^{2} d s+C(t, 0)\left(\int_{0}^{t} g(u, z(u)) d u\right)^{2}
$$

and $C$ convex satisfies

$$
z(t)=a(t)-\int_{0}^{t} C(t, s) g(s, z(s)) d s
$$

$$
\begin{aligned}
V^{\prime}(t) \leq & 2 g(t, z) \int_{0}^{t} C_{s}(t, s) \int_{s}^{t} g(u, z(u)) d u d s+2 g(t, z) C(t, 0) \int_{0}^{t} g(u, z(u)) d u \\
= & 2 g(t, z)\left[\left.C(t, s) \int_{s}^{t} g(u, z(u)) d u\right|_{0} ^{t}+\int_{0}^{t} C(t, s) g(s, z(s)) d s\right] \\
& +2 g(t, z) C(t, 0) \int_{0}^{t} g(u, z(u)) d u \\
= & 2 g(t, z) \int_{0}^{t} C(t, s) g(s, z(s)) d s=2 g(t, z)[a(t)-z(t)] .
\end{aligned}
$$

When $g(t, z)=z$, this readily yields

$$
V^{\prime}(t) \leq a^{2}(t)-z^{2}(t)-(z(t)-a(t))^{2},
$$

as used in Section 2.

For the lower bound,

$$
\begin{aligned}
(z(t)-a(t))^{2} & =\left(\int_{0}^{t} C(t, s) g(s, z(s)) d s\right)^{2} \\
& =\left(-\left.C(t, s) \int_{s}^{t} g(u, z(u)) d u\right|_{0} ^{t}+\int_{0}^{t} C_{s}(t, s) \int_{s}^{t} g(u, z(u)) d u\right)^{2} \\
& \leq 2\left[\left(C(t, 0) \int_{0}^{t} g(u, z(u)) d u\right)^{2}+\left(\int_{0}^{t} C_{s}(t, s) \int_{s}^{t} g(u, z(u)) d u\right)^{2}\right]
\end{aligned}
$$




\section{THEODORE A. BURTON}

$$
\begin{aligned}
\leq & 2\left[C^{2}(t, 0)\left(\int_{0}^{t} g(u, z(u)) d u\right)^{2}\right. \\
& \left.+\int_{0}^{t} C_{s}(t, s) d s \int_{0}^{t} C_{s}(t, s)\left(\int_{s}^{t} g(u, z(u)) d u\right)^{2} d s\right] \\
\leq & 2\left[C(t, 0)+\int_{0}^{t} C_{s}(t, s) d s\right] V(t) \\
= & 2\left[C(t, 0)+\left.C(t, s)\right|_{0} ^{t}\right] V(t)=2 C(t, t) V(t) .
\end{aligned}
$$

\section{REFERENCES}

[1] BURTON, T. A.: Boundedness and periodicity in integral and integro-differential equations, Differential Equations Dynam. Systems 1 (1993), 161-172.

[2] BURTON, T. A.: Liapunov Functionals for Integral Equations, Trafford, Victoria, B. C., Canada, 2008, (www.trafford.com/08-1365).

[3] BURTON, T. A.: A Liapunov functional for a singular integral equation, Nonlinear Anal. 73 (2010), 3873-3882.

[4] BURTON, T. A.-DWIGGINS, D. P.: Resolvents, integral equations, and limit sets, Math. Bohem. 135 (2010), 337-354.

[5] BURTON, T. A.-DWIGGINS, D. P.: Smoothed integral equations, J. Math. Anal. Appl. 377 (2011), 319-335.

[6] MILlER, R. K.: Nonlinear Volterra Integral Equations, W. A. Benjamin, Menlo Park, CA, 1971.

[7] MILlER, R. K.-NOHEL, J. A.-WONG, J. S. W.: Perturbations of Volterra integral equations, J. Math. Anal. Appl. 25 (1969), 676-691.

[8] ISLAM, M. N.-NEUGEBAUER, J. T.: Qualitative properties of nonlinear Volterra integral equations, Electron. J. Qual. Theory Differ. Equ. 12 (2008), 1-16.

[9] STRAUSS, A.: On a perturbed Volterra integral equation, J. Math. Anal. Appl. 30 (1970), 564-575.

[10] VOLTERRA, V.: Sur la théorie mathématique des phénomès héréditaires, J. Math. Pur. Appl. 7 (1928), 249-298.

Received August 7, 2010

Northwest Research Institute

732 Caroline St.

Port Angeles

Washington 98362

U.S.A.

E-mail: taburton@olypen.com 